\title{
DOCUMENTATION
}

\section{LES NOUVELLES RACES PORCINES AMÉRICAINES}

PAR

\section{R. FEVRIER et P. VIEILLART}

Station de Recherches sur l'Élevage Centre National de Recherches Zootechniques

En I945, les communications normales étant rétablies avec le Nouveau Monde, les zootechniciens européens apprenaient, par des informations encore fragmentaires, que l'on créait aux U. S. A. plusieurs nouvelles races de porcs : Minnesota $n^{\circ} I$, Minnesota $n^{\circ} 2$, Hamprace. Ces renseignements devinrent plus abondants par la suite : l'attrait de la nouveauté et des produits américains aidant, le public prêta volontiers à ces races nombre de qualités, et des demandes d'importation furent présentées à plusieurs administrations européennes.

L'objet de cette mise au point est d'apporter quelques précisions sur ce sujet, d'exposer le but poursuivi par leurs créateurs, de décrire les méthodes employées et d'étudier les résultats obtenus.

\section{LE BUT}

En créant ces races, les chercheurs américains ne voulaient pas ${ }^{(1)}$ uniquement ajouter quelques unités de plus à la gamme ${ }^{(2)}$ déjà étendue des races porcines exploitées aux U.S. A. Une telle variété, loin d'être une richesse, constitue souvent un danger en provoquant la dispersion des efforts de sélection et en multipliant les causes d'indésirables métissages, elle est redoutée aussi bien par CARRoll et KRIDER (7) aux U. S. A. que par Allix (I-2) et QUITTET (42) en France et par CROWTHER (14) en Angleterre. Leur but était d'obtenir un porc destiné à être croisé, d'après WINTERS et ses coliaborateurs (5), soit indifféremment avec d'autres races (Minensota $n^{0} I$ ), soit plus spécialement avec l'une d'elles (Minnesota $n^{0} 2$ ) (46), plutôt que d'être utilisé directement pour la production de la viande.

\section{L'intéret du croissement}

La supériorité des animaux issus d'un croisement est, en effet, l'idée directrice de la plupart des études réalisées ces dernières années aux U.S. A. et le succès des maïs hybrides n'a pas été sans influencer les tendances de la sélection animale dans ce Pays, où l'on pense produire en I95I 30 à 40 millions de " poulets hybrides".

ROBERTS et CARROLL, après avoir comparé par la méthode de la double-saillie $\left({ }^{3}\right)$ des animaux de pure race et des animaux croisés $(43)$ et après avoir examiné les résultats expérimentaux portant sur plus de 50000 animaux (6), restent sceptiques sur l'intérêt systématique que peut présenter le croisement. RoBIson (44), sans nier les résultats parfois négatifs des

(1) Du moins pour les races Minnesota $n^{\circ} 1$ et 2. L'objectif des créateurs de la Hamprace semble avoir été plus classique.

(2) D'après BRIGGS (4) : Berkshire - Hampshire - Poland China - Spotted Poland China Duroc Jersey - Chester White - Yorkshire Large White - Tamworth - Hereford.

(3) Une truie est successivement saillie par deux verrats dont les descendants éventuels peuvent être facilement distingués (oreilles, pelage, etc.). 
études réalisées, estime qu'ils dépendent des animaux utilisés et que sélection et pratique du croissement ne s'excluent pas. Lush et al. (38-39), à la suite de longues études au cours desquelles ils utilisent la méthode de la double-saillie, expriment également une opinion nuancée : l'animal croisé n'est pas toujours statistiquement supérieur au meilleur parent pour chaque caractère, mais si l'on considère l'ensemble des caractères, il manifeste générale:nent sa supériorité. DICKERSON (I9) va plus loin : chacune des races existantes manifeste une supériorité pour une partie des caractères utiles, mais les croisés égalent ou dépassent la meilleure des races dont ils sont issus pour chaque caractère, et par là possèdent un net avantage global. Whiteman, Hillier, Whatley (56) notent des différences dans la carcasse en faveur des porcs croisés. Mais les échantillons étudiés sont réduits, et les différences sont peu significatives.

Quant au créateur des nouvelles races, WinTERs, après avoir comparé pendant 6 ans des animaux de race pure et issus de croisements de trois types : crossbred $\left.{ }^{(}\right)$, three bred $\operatorname{cross}\left({ }^{2}\right)$ backcross $\left({ }^{3}\right)(58)$, il affirme (59) : "Les résultats obtenus dans les troupeaux expérimentaux dans les fermes montrent que, pour la production de porcs destinés à l'abattage, les trois types de croisements possèdent de nets avantages. "Et plus tard, à la suite d'une étude de l'hétérosis (48), il précise : "L'augmentation de vigueur résultant d'un croisement de deux souches (strains) $\left({ }^{4}\right)$ consanguines (inbred) de maïs ou d'animaux de laboratoire est un fait établi. "

\section{Role de la consanguinite}

L'effort des Stations de recherches, dont l'activité est coordonnée depuis I937 par le Regional Swine Laboratory (I3) de AMEs (Iowa), a porté, dans le domaine de la sélection, vers la création de lignées consanguines (inbred lines) $\left({ }^{5}\right)$ pour permettre des croisements (linecross) dont on attenda it des résultats meilleurs qu'en croisant des animaux de races différentes (crossbred).

La plupart des auteurs trouvent, en effet, une supériorité des produits de croisements entre lignées inbred (line cross), sensible surtout en ce qui concerne le nombre de porcelets élevés, quoique, dans une étude portant sur 800 portées de 50 fermes de I'Iowa, HAZEL, Husson et LUSH (23) n'aient pas observé de différence significative entre les résultats obtenus par l'emploi de verrats inbred et de verrats ordinaires de race pure Poland China. DuRHAM (20) confirme ce résultat en étudiant les troupeaux de 18 fermes du Wisconsin. Mais HeTzer et ses collaborateurs (25) constatent une diminution nette de la mortalité en croisant entre elles des lignées inbred ( $29 \%$ à 56 jours) ; par contre, le poids de l'animal n'est augmenté que de $5 \%$ à 140 jours et l'amélioration de la carcasse est insignifiante $(r, 5 \mathrm{~mm}$ de lard en moins). WARWICK et WILEY (54) ne mettent pas en évidence de nettes améliorations dans la croissance des porcs croisés, après avoir comparé pendant 3 ans des animaux purebred, crossbred et crossline ; seul le poids à 56 jours fut généralement plus élevé chez les "crossline ". Dickerson, Lush et CulberTSON ( 17 ) trouvent, au sevrage, une amélioration de $\mathrm{I}, 6$ porcelet par portée et de de $3,6 \mathrm{lb}$ par animal entre les crossline et les inbred line. CHAMBERS et WHATLEY (9), dans un travail analogue, trouvent 0,88 porcelet de plus par portée. SIERK (47), travaillant sur 373 portées issues de croisements entre lignées inbred, constate une amélioration de vigueur $\left({ }^{6}\right)$ de 4 à I I \% par rapport aux inbred dans les différents croisements qu'il opère (Poland China $\times$ Minnesota $n^{\circ}$ I ou 2 , Minnesota $n^{\circ} I \times$ Minnesota $n^{0} 2$ ) et qui atteint $15 \%$ par rapport à des croisements entre non inbred.

\section{L'inbreeding abaisse la vitalité des animaux}

C'est là, en effet, toute la question : que les line cross manifestent une " vigueur "supérieure aux inbred, cela semble établi. Mais il ne convient pas d'oublier que l'établissement de souches inbred s'accompagne d'une diminution des qualités utiles et que, pour s'avérer rentable, le linecrossing doit provoquer une amélioration supérieure à la perte subie lors de la création des souches parentes.

Sur les Végétaux, le phénomène est bien connu et l'affaiblissement des souches inbred de mais en constitue un exemple classique (33).

Nous employons les termes anglo-saxons quand il peut y avoir ambiguité $\left(3^{2}\right)$ :

(1) Crossbred - produits du croisement de 2 races différentes.

(2) Three bred cross - produits du croisement de 3 races différentes. Ex. $(\mathrm{A}+\mathrm{B}) \times \mathrm{C}$.

(3) Back-cross - produits du croisement d'un crossbred avec un sujet d'une race parente.

(4) Inbred line - ensemble d'animaux provenant de sujets apparentés entre eux.

(5) Inbred strain - "inbred line " où les sujets ont un coefficient d'inbreeding supérieur à 35,7 \%.

Nous traduirons les deux termes "line " et "strain " par souche. mation.

${ }^{(6)}$ Les comparaisons portent sur le poids au sevrage, la vitesse de croissance, l'indice de consom- 
Sur des animaux de laboratoire, KING (30) n'avait pas noté de sensible perte de vigueur au bout de 25 générations successives de rats obtenus par le croisement frère $\mathrm{x}$ sœur, mais WRIGHT (64) avait constaté un déclin de vitalité sur des souches inbred de cobayes.

Sur des animaux domestiques, Hughes (27) avait constaté une légère diminution de l'importance des portées dans des familles de Berkshire inbred, alors que GODBEY et STARKEY (22) observaient un déclin plus sensible. HoDGSON (26), en 1935, avait constaté chez le porc une diminution de la vitesse de croissance et une réduction du nombre des porcelets par portée, qu'il attribuait cependant à la brutalité accrue de certaines mères, autant qu'au manque de vigueur de leurs petits. BERGE (3) montre, sur le Large White et la race porcine norvégience, un affaiblissement des performances à mesure que le coefficient d'inbreeding croît. McPHEE, Russel et des ZELler (40) et LuSh et CUlBERTSON (36) constatent une forte diminution aptitudes utiles en pratiquant de l'inbreeding pendant plusieurs générations de Poland China. Le même résultat est obtenu sur des Duroc-Jersey par WilhaM et CRAFT (57).

Dickerson et Lush ( 7 - 78 ) calculent qu'à chaque augmentation de ro $\%$ du coefficient de consanguinité, il y a une diminution hautement significative d'environ 0,7 porcelet élevé par portée et de 3,4 lb à 5 mois par animal. Ils remarquent également une influence défavorable sur l'utilisation de la nourriture et les qualités de la carcasse. Il convient donc de remédier à ce danger par la sélection, mais CoMSTOCK et WINTERS ( $\mathrm{r} 2$ ) estiment qu'une forte sélection permet de contrebalancer les effets de $15 \%$ d'inbreeding par génération pour la vitesse de croissance, et de $2,5 \%$ pour la fertilité, ce qui explique les difficultés rencontrées sur ce point. Plus tard, Winters, Cummings et Stewart (62), étudiant leur méthode d'inbreeding " souple " (c'est-à-dire sans croisement systématique entre sujets ayant un degré donné de parenté tel que frère et sœur), constatent que la sélection leur a permis de maintenir les différentes qualités des animaux sans baisse significative, malgré un très fort coefficient d'inbreeding; mais KoTTMAN, LUSH et HAZEL (3I) voient le niveau des performances baisser dans le troupeau de l'Université de l'Iowa, les effets de l'inbreeding l'emportant sur ceux de la sélection.

\section{Comparaison linecross-outbred}

Il importe donc de comparer les linecross, moins aux parents inbred qu'aux animaux issus des méthodes classiques de sélection. WINTERS et ses collaborateurs $\left(6_{3}\right)$ disent très justement : "L'objectif final du développement des souches inbred est leur emploi dans des croisements pour la production de porcs destinés à l'abattage. Pour cette raison, la valeur d'une lignée inbred ne peut être appréciée ni sur un point particulier, ni globalement, par ses propres performances comme inbred. is

Le travail de Chambers et Whatuey (8-9) poursuivi de 1938 à 1945, nous apporte sur ce point d'intéressantes précisions. Travaillant sur 7 lignées inbred, ces auteurs comparent les animaux issus de croisements entre 2 ou 3 de ces lignées, aux animaux des lignées parentes et aux animaux ordinaires. Les résultats sont présentés dans le tableau suivant, qui exprime les différences observées lors des diverses comparaisons. (Exemple : dans la comparaison I, le croisement de 2 souches inbred a donné, par rapport à la souche de la femelle, 0,43 porcelet vivant de plus, $0,55 \mathrm{~kg}$ de plus par portée à la naissance, etc. Dans la comparaison V, le croisement de 2 souches inbred a donné, par rapport à des Duroc-Jersey ordinaires, 0,92 porcelet de moins à la naissance, 0,39 porcelet de moins à 21 jours, 0,28 porc de moins à 56 jours, etc.)

\begin{tabular}{|c|c|c|c|c|c|c|}
\hline Nature de la comparaison: & 1 & II & III & IV & & $\mathrm{V}$ \\
\hline Naissance: & & & & & & \\
\hline de porcelets vivants par portée... & 0,43 & 0,48 & $\mathrm{x}, 36$ & 1,17 & - & 0,92 \\
\hline de la portée $(\mathrm{kg}) \ldots \ldots \ldots \ldots \ldots \ldots$ & 0,55 & 0,76 & 1,33 & $\mathrm{I}, 24$ & - & $0,9 \mathrm{I}$ \\
\hline de porcelets. & 0,76 & 0,74 & I, I 5 & 1,60 & - & 0,39 \\
\hline de la portée $(\mathrm{kg}) \ldots \ldots \ldots \ldots \ldots \ldots$ & $3,3^{6}$ & 3,96 & $4,3^{2}$ & 5,89 & - & 2,40 \\
\hline de porcelets................ & 0,85 & 0,88 & $\mathrm{I}, 20$ & 1,49 & - & 0,28 \\
\hline 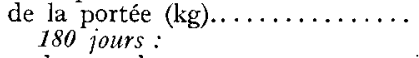 & 10,64 & I 3,68 & 13,44 & $\mathrm{II}, 2 \mathrm{O}$ & - & 6,04 \\
\hline $\begin{array}{l}\text { re de porcelets..... } \\
\text { de la portée }(\mathrm{kg}) . .\end{array}$ & I, 08 & $\begin{array}{r}1,31 \\
106\end{array}$ & I,66 & $\begin{array}{r}2,19 \\
-8\end{array}$ & 一 & 0,35 \\
\hline & 81,94 & 100,45 & 134,90 & 158,0 & - & $6, I_{1}$ \\
\hline
\end{tabular}

Comparaison :

I croisement de 2 souches inbred - souche de la $q$
II
III 
On peut remarquer que le croisement de 2 lignées inbred donne des résultats nettement supérieurs à ceux des lignées originelles, légèrement inférieurs à ceux des porcs non inbred, lesquels sont surclassés uniquement par le produit de croisement de 3 lignées inbred. (Cette supériorité est significative pour le nombre et le poids des porcelets nés vivants).

Sans mettre en doute les conclusions de ces travaux, qui ont requis une continuité et une amplitude dans l'effort qu'il convient de souligner $\left(^{(1)}\right.$, il nous paraît important de noter que les résultats obtenus par ces différents moyens sont pour le moins médiocres en valeur absolue, et que le résultat des comparaisons ne doit pas faire oublier le niveau auquel elles sont opérées. C'est ainsi que WARWICK et WILEY (54) donnent, comme nombre de porcelets sevrés chez les animaux "améliorés " par les méthodes classiques : 5,2 par portée, et que DicKERSON, LUSH et CULBERTSON ( 17 ) obtiennent 3 porcelets par portée inbred et 4,8 par portée linecross à $r 54$ jours. Chambers et WhatLey (9) obtiennent à I 80 jours, par portée : 4,6 avec les inbred, 5,7 avec le croisement de 2 lignées inbred, 5,7 avec les porcs normaux et 6,9 pour ceux obtenus à partir de 3 lignées inbred. Ces résulats sont faibles, même comparés à la valeur moyenne obtenue aux U.S. A. et qui est de l'ordre de $6,25(52)$ déjà très faible en elle-même.

Dans le cadre de ce projet concernant la création de souches consanguines et désirant obtenir la meilleure combinaison possible de gènes, les zootechniciens américains imaginèrent de créer des races, ou plus exactement des souches (lines) à partir de plusieurs races dont ils voulaient associer les caractères. Laissons la parole à WINTERS, CoMSTOCK et DAILEY (60), $\grave{a}$ propos de la race Minnesota $n^{0} I_{\text {. }}$ "Les objectifs de cette méthode sont:

$\mathrm{I}^{\circ}$ de combiner dans un seul "strain" un maximum de caractères utiles appartenant aux deux races mères;

$2^{\circ}$ de provoquer une recombinaison de facteurs génétiques d'oì résulterait l'extériorisation de nouveux caractères désirables non visibles chez les parents. En l'occurrence, le but était de combiner au maximum la longueur du corps et le rebondi du jambon du porc danois avec la couleur rouge, la prolificité et la valeur laitière du Tamworth et en même temps, si possible, de provoquer, par la combinaison des gènes, une accumulation d'autres caractères désirables des deux races.

Johansson (29) a dressé le tableau des différentes lignées consanguines obtenues à partir d'un croisement :

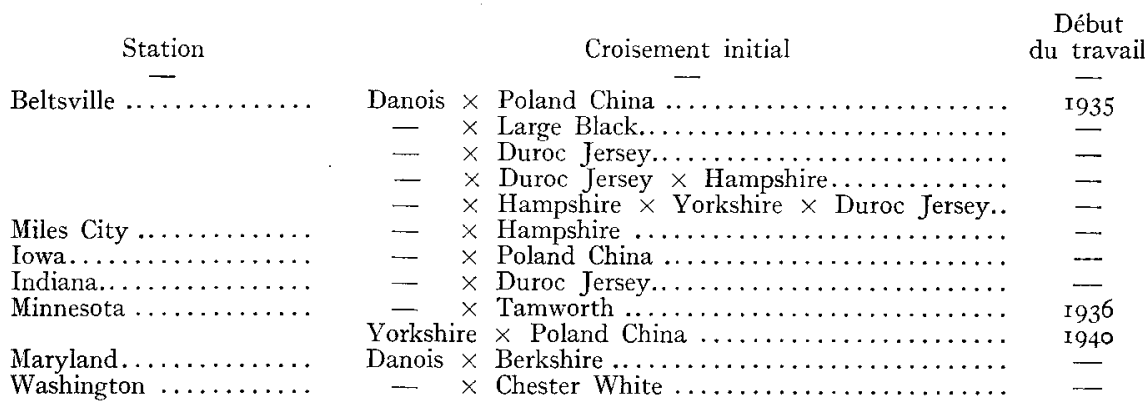

Celles qui, actuellement, semblent avoir le plus d'importance ont été produites aux Stations du Minnesota, et de Miles City. Elles ont été nommées Minnesota $n^{0} x$, Minnesota $n^{0} 2$, et Hamprace. Des livres généalogiques ont été créés pour chacune d'elles.

\section{LES METHODES}

\section{Minnesota $\mathrm{n}^{\circ} \mathbf{1}$}

Il provient d'un croisement effectué en 1937 du verrat danois I8-2 avec 6 truies Tamworth. Nous avons déjà vu les raisons qui ont guidé le choix des auteurs, vers ces deux races ; il convient d'ajouter que l'origine étrangère de ces races permettait d'espérer, lors d'un croisement ultérieur avec des animaux américains, un plus fort degré d'hétérosis, comme le pensent Hazel, Husson et Lush (23) et Stewart et Comstock (50).

(1) Au début de 1947 , ces recherches sur les souches inbred avaient utilisé 74 II r portées comprenant $5^{8} 822$ porcelets à la naissance, d'après JoHansson (29). 
Le verrat provenait de la Station fédérale de Beltsville, qui avait importé en I934 du Danemark un certain nombre de reproducteurs de la célèbre Danish Landrace. Les truies provenaient, 3 du l'Iowa State College, et 3 de l'Université du Saskatchevan. Les six portées nées furent contrôlées pour le poids et le nombre des porcelets au sevrage, et la quantité de nourriture consommée à I 54 jours ; de ce fait, une fut éliminée et desautres, I 3 femelles et 3 mâles furent conservés pour la reproduction.

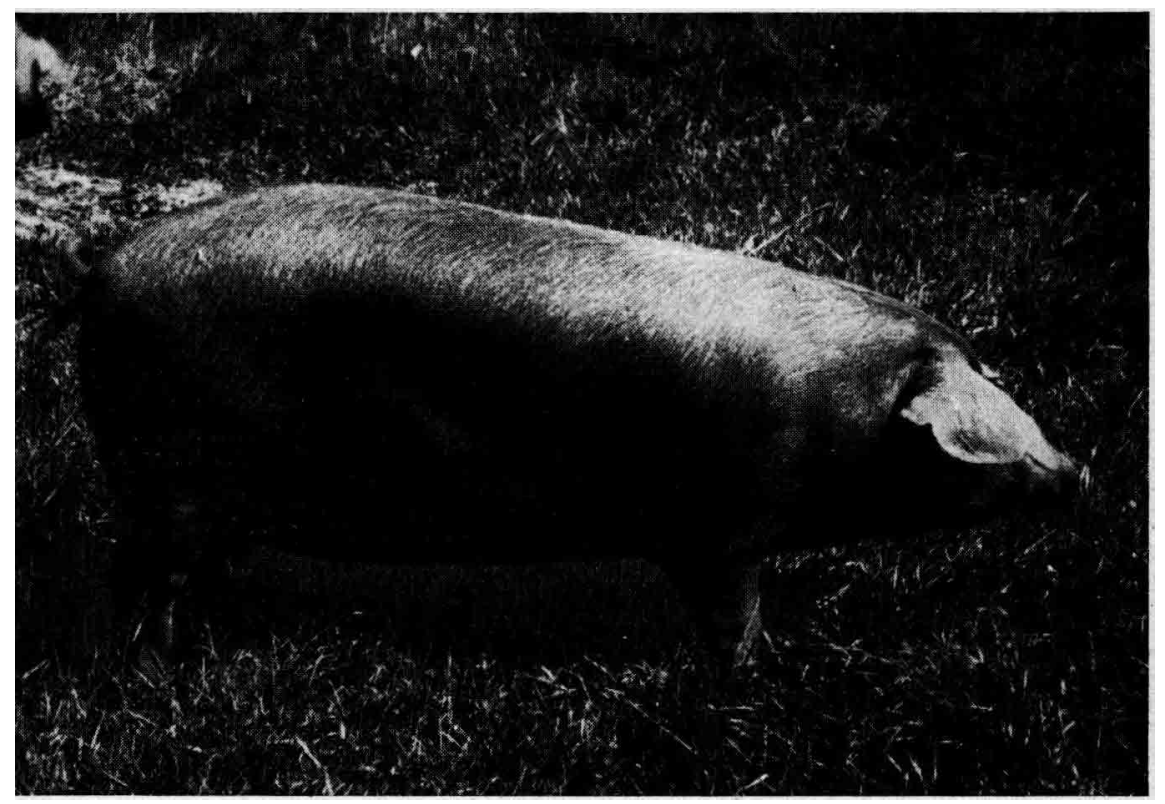

Porc Minnesota no I. (Cliché Professeur Winters)

En 1938 , on introduisit dans le troupeau:

2 nouvelles truies Tamworth.

4 nouvelles truies Danoises.

I nouveau verrat Danois.

et le plan des saillies fut le suivant :

les $I_{3}$ femeiles $F_{1}$ furent saillies par les 3 verrats $F_{1}$.

$\begin{array}{r}5 \\ -4\end{array}-$ Tamworth - - Danoises - - -

- ${ }_{\mathrm{I}}-\mathrm{C}$ Tamworth fut saillie par l'ancien verrat danois

- I - $\quad-\quad$ par le nouveau verrat $\underset{6-7}{\mathrm{I} 8-2}$

Ensuite, furent vendus tous les animaux de pure race et les produits de la $F_{1}$. La sélection fut limitée aux portées de 1938 et fut effectuée d'après les tests que nous avons décrits. Il est important de souligner, comme le font les auteurs, que l'on n'a pas' appliqué un plan rigide de croisement ; cette méthode aurait pu avoir comme effet d'interdire certaines alliances intéressantes, ce qui peut expliquer certains échecs rencontrés dans les tentatives précédentes d'inbreeding (6I). Ce sont les qualités des sujets qui, à l'intérieur du groupe des animaux de 1938, permirent de déterminer les croisements à opérer : "Les meilleurs furent alliés aux meilleurs". Une attention toute particulière fut attachée à la fertilité, qui est probablement, nous l'avons vu, le facteur le plus sensible à l'inbreeding. Aussi, à partir de r 939 , on n'hésita pas à produire 30 à $50 \%$ plus de portées que l'on ne pouvait en contrôler, de façon à pouvoir éliminer avec sévérité les familles manquant de fertilité (60).

Annales de Zootechnie. - $195^{2}$ 
Les critères utilisés dans le choix des reproducteurs furent, par ordre d'importance : fertilité,

survie (\% de sevrés),

vitesse de croissance,

indice de consommation,

La sévérité de la sélection est indiquée par le tableau suivant, que nous empruntons à Brugman, Winters et Dailey (5):

$\begin{array}{lllllllllll}1937 & 1938 & 1939 & 1940 & 1941 & 1942 & 1943 & 1944 & 1945 & 1946 & \text { Moy. }\end{array}$

Total des portées nées.... Portées testées \% ...... $\%$ des animaux sevrés en animaux testés ..... en animaux conservés pour la reproduction... en animaux ayant de la descendance (déc. 48)..

\begin{tabular}{|c|c|c|c|c|c|c|c|c|c|c|}
\hline $\begin{array}{c}6 \\
100,0\end{array}$ & $\begin{array}{c}24 \\
100,0\end{array}$ & $\begin{array}{c}39 \\
53,8\end{array}$ & $\begin{array}{l}-\overline{42} \\
64,3\end{array}$ & $\begin{array}{c}\overline{45} \\
53,3\end{array}$ & $\begin{array}{c}\overline{43} \\
60,5\end{array}$ & $\begin{array}{c}\overline{49} \\
53, \mathrm{I}\end{array}$ & $\begin{array}{c}-\overline{4 I} \\
5^{6, I},\end{array}$ & $\begin{array}{c}\overline{5^{2}} \\
4^{8, \mathrm{I}}\end{array}$ & $\begin{array}{c}\overline{46} \\
43,5\end{array}$ & $\begin{array}{r}\overline{387} \\
57,4\end{array}$ \\
\hline 100,0 & 96,7 & 45,5 & $7 \mathrm{I}, 7$ & $6_{3, I}$ & 70,6 & 75,3 & $4 \mathrm{I}, 5$ & 63,0 & 46,0 & 63,2 \\
\hline 33,3 & 23,6 & $I_{4}, 5$ & $\mathrm{I} 2,3$ & I0,8 & I 2,9 & 7,3 & $I 4,7$ & I0,8 & 17,9 & 13,7 \\
\hline I6,7 & 5,5 & 4,0 & 4,8 & 3,6 & 5,3 & 4,2 & 6,8 & 6,7 & 20,4 & 6,8 \\
\hline
\end{tabular}

Nous soulignons à nouveau l'importance des moyens employés : pour cette seule étude une quarantaine de portées furent nécessaires à chaque génération.

Si l'on examine l'influence dans le troupeau actuel (1948) des animaux de départ, on constate $(5)$ que le $2^{\mathrm{e}}$ verrat danois, qui paraissait supérieur au $\mathrm{r}^{\mathrm{er}}$, ne joue pas de rôle sensible dans le patrimoine héréditaire des animaux actuels :

Animaux d'origine

Coefficient de parenté avec les animaux actuels

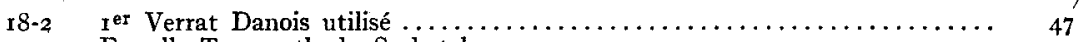

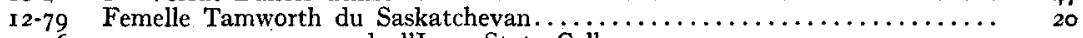

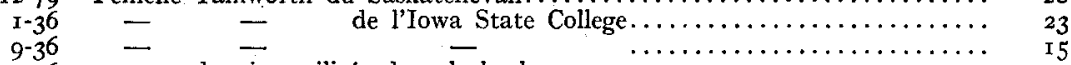

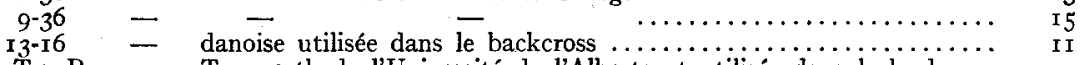

T-74R - Tamworth de l'Université de l'Alberta et utilisée dans le backcross 0,05

En 1947, on pouvait estimer que les gènes provenaient, pour $48 \%$ des porcs danois et pour $52 \%$ des Tamworths. Le coefficient d'inbreeding calculé d'après la formule de WRIGHT $(65-66)$ Fx était de 0,36 en I948, mais grâce à la méthode employée (sélection sévère et eroisements souples), les qualités des animaux ne sont pas tombées aussi bas qu'on pouvait le craindre :

\begin{tabular}{|c|c|c|c|c|c|c|}
\hline & 1937 & 1988 & 1939 & 1940 & 1941 & 1942 \\
\hline Fx des portées. & 0,00 & 0,07 & 0,14 & $\overrightarrow{0,19}$ & 0,22 & \\
\hline Nombre de moyen porcelets nés vivants & 9,2 & 9,5 & $\begin{array}{l}0,14 \\
8,8\end{array}$ & 9,3 & $\begin{array}{l}0,22 \\
8,6\end{array}$ & $\begin{array}{l}0,24 \\
8,8\end{array}$ \\
\hline Nombre moyen de porcelets sevrés ... & 8,0 & 7,7 & 7,1 & 7,0 & 6,8 & $7, \mathrm{I}$ \\
\hline Poids au sevrage $(\mathrm{kg}) \ldots \ldots \ldots \ldots \ldots$ & I0,9 & 15,4 & I. 3,6 & $13, I$ & 15,9 & I 4,9 \\
\hline 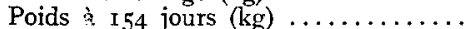 & 86,5 & 80,6 & 82,0 & 88,3 & 83,4 & 87,9 \\
\hline Gain moyen quotidien ............ & 0,69 & 0,68 & 0,71 & 0,76 & 0,68 & 0.74 \\
\hline \multirow{3}{*}{ Indice de consommation (au pâturage) } & 3,08 & 3,58 & 3, I 5 & 3,02 & 3,07 & 3,06 \\
\hline & 1943 & 1944 & 1945 & 1946 & 1947 & 1948 \\
\hline & - & 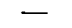 & - & - & - & \\
\hline Fx des portées. & 0,27 & 0,29 & 0,29 & 0,32 & 0,34 & 0,36 \\
\hline Nombre moyen de porcelets nés vivants & 9,8 & 10,2 & 9,6 & 9,65 & 8,26 & 9,2 \\
\hline Nombre moyen de porcelets sevrés ... & 7,0 & 7,0 & 6,6 & 6,8 & 3,97 & 6,8 \\
\hline Poids au sevrage $(\mathrm{kg}) \ldots \ldots \ldots \ldots \ldots$ & I 3,6 & 14,0 & 14,0 & $\mathrm{Ir}, 3$ & 12,0 & 12,4 \\
\hline Poids à r 54 jours $(\mathrm{kg}) \ldots$ & 75,2 & 78,8 & 84,3 & 83,4 & 77,0 & 71,1 \\
\hline Gain moyen quotidien ............ & 0,62 & 0,66 & 0,72 & 0,70 & 0,65 & 0,59 \\
\hline Indice de consommation (au pâturage). & 3,13 & 3,08 & $3, \mathrm{II}$ & 3,08 & 2,84 & 2,94 \\
\hline
\end{tabular}

En 1946, le Minnesota $n^{\circ} I$ pouvait être enregistré sur les livres de "The Inbred Livestock Registry Association " qui venait de se former.

Le porc Minnesota $n^{\circ} I$ actuel a un corps qui rappelle un peu le Danois, quoique moins fin, et moins long. Il est plus long que les porcs américains et sa conformation nous paraît meilleure. Sa robe est celle du Tamworth, rouge bistre. 


\section{Minnesota $\mathrm{n}^{0} 2$}

Il fut créé à Crookston par la même équipe de chercheurs et toujours en coopération avec le Regional Swine Laboratory. Leur but, disent-ils, était la création d'un type de porc maigre qui pourrait être croisé avec le Minnesota $n^{0} I$.

La même méthode fut utilisée ( flexible system "avec sélection rigoureuse), à partir dc I 941, mais on prêta davantage attention aux caractères n'affectant pas directement l'utilité (fancy points), de façon à créer une race différant le plus possible du Minnesota $n^{0} I$ par son aspect extérieur. "On pensait, en effet, qu'en développant deux lignées qui posséderaient en commun de nombreux caractères économiques, mais différeraient autant que possible par leurs caractères superficiels, on augmenterait les chances de réussite de leur croisement (63)."

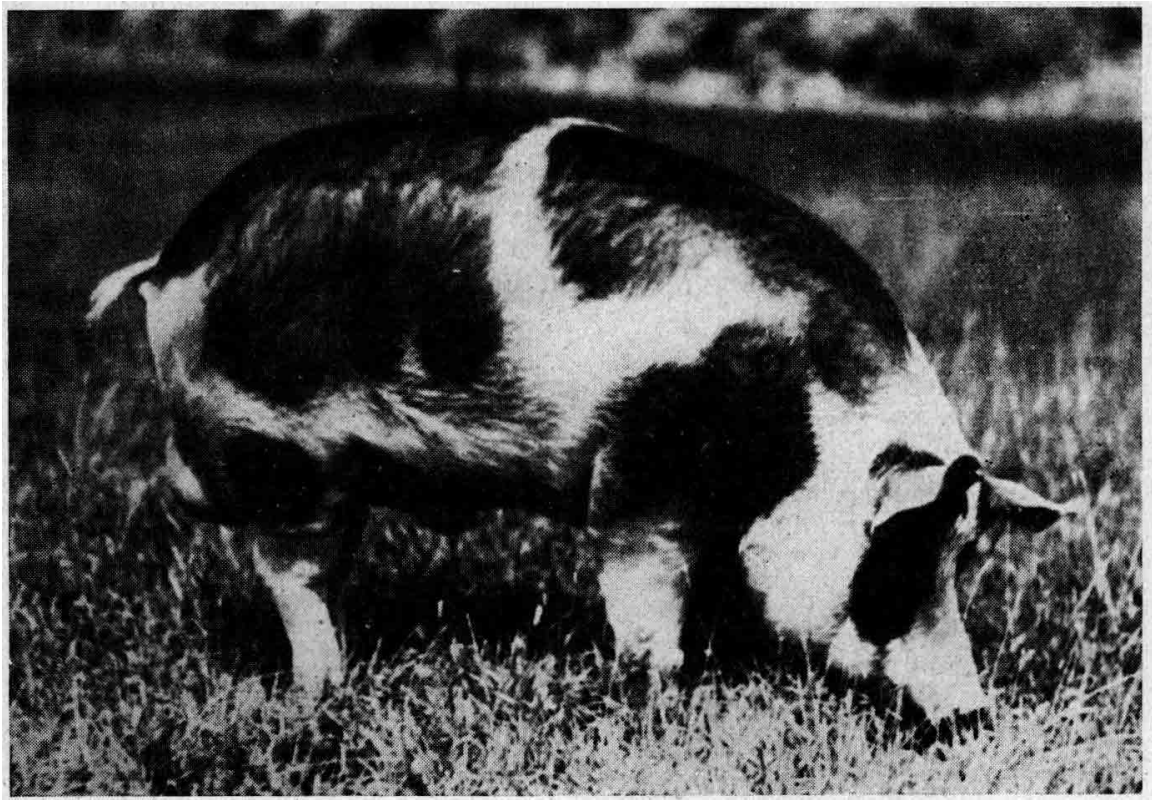

Porc Minnesota $\mathrm{n}^{\circ} 2$ (Cliché Professeur Winters)

Les animaux désirés étant destinés à être croisés aux Minnesota $n^{\circ}$, il était souhaitable de fonder cette race avec des animaux ayant une origine différente. Des épreuves antérieures avaient montré que deux lignées inbred de Poland China (5I) Market Lady et Black Star) possédaient une bonne croissance, un faible indice de consommation et produisaient des carcasses satisfaisantes. Cependant, les truies manquaient de fertilité et étaient de mauvaises laitières. On pensa améliorer la production laitière et la fertilité des truies en utilisant le Yorkshire canadien (5I).

En I94I, le verrat Yorkshire canadien $7 \mathrm{~V}$ fut croisé avec 13 truies Poland China. Les porcs de la $F_{1}$ et de la $F_{2}$ ne furent pas satisfaisants, ni dans leurs performances, ni dans leur type. Aussi, à l'automne 1943 , la moitié des femelles de la $\mathrm{F}_{2}$ fut recroisée (backross) avec des verrats des mêmes lignées de Poland China. Le type et les performances furent améliorés.

Comme pour le Minnesota $n^{\circ} I$, une grosse élimination fut effectuée, basée sur la qualité des individus et de leur descendance.

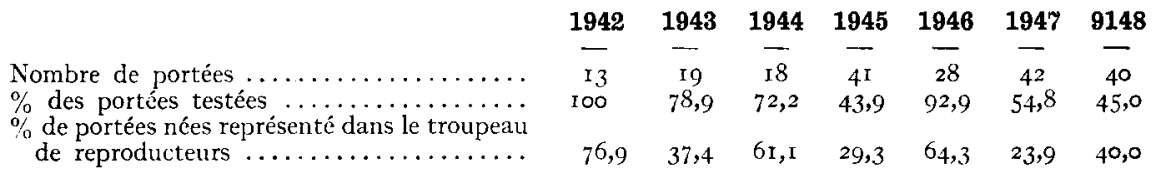


En I948, bien que le coefficient de consanguinité fut de 0,28 , les performances des animaux ne semblaient pas avoir trop baissé depuis le début ; on peut estimer que les animaux actuels possèdent $40 \%$ de gènes Yorkshire et $60 \%$ de gènes Poland China.

\begin{tabular}{|c|c|c|c|c|c|c|c|}
\hline & 1942 & 1943 & 1944 & 1945 & 1946 & 1847 & 1948 \\
\hline & & & & & - & & \\
\hline Fx (coef. de consanguinité) & - & 0,20 & 0,16 & 0,19 & 0,23 & 0,24 & 0,28 \\
\hline Nés par portée .......... & 6,2 & $9, \mathrm{I}$ & 8,5 & 8,5 & 8,0 & 7,6 & 7,5 \\
\hline Sevrés par portée. & 5,5 & $6, I$ & 4,4 & 4,7 & 3,8 & $4, I$ & 6,1 \\
\hline Poids au sevrage $(\mathrm{kg})$ & $\pm 3,6$ & 10,6 & 14,4 & 15,8 & 14,4 & 13,6 & 13,2 \\
\hline Poids à I 54 jours $(\mathrm{kg})$ & 75,6 & 61,6 & 80,6 & 84,7 & 77,0 & 69,3 & 73,8 \\
\hline Indice de consommation (au pâturage). & 3,08 & $2,8 \mathrm{I}$ & 3,55 & $3, \mathrm{ir}$ & $3,3^{6}$ & 3,16 & 2,94 \\
\hline
\end{tabular}

En 1943 , un $2^{\mathrm{e}}$ troupeau de Minnesota $n^{0} 2$ fut créé à la Northeast expériment station à Duluth (Minnesota). Un verrat Poland China de la lignée C, provenant de la station centrale de Saint-Paul (Minnesota) fut croisé avec 4 fernelles Minnesota $\mathrm{n}^{\circ} 2$ provenant du troupeau de Crookston. La même méthode de croisement et de sélection fut suivie et seules quelques introductions de reproducteurs provenant de Crookston furent opérées. Dans ce troupeau, le coefficient de consanguinité atteignait $0,3 \circ$ en 1948 et les performances paraissent meilleures que celles de Crookston ( 8,9 et 8 porcelets nés et sevrés par portée, $\mathrm{I3}, 6 \mathrm{~kg}$ de moyenne au sevrage).

Le porc ainsi obtenu a l'allure d'un Yorkshire, près de terre et court. Ses oreilles sont droites, légèrement penchées en avant. Sa robe est blanche, avec des taches noires assez importantes, couvrant parfois la plus grande partie du corps.

\section{Hamprace ou montana $n^{0} 1$}

Il a été créé dans le Montana (U. S. Range Livestock Experiment Station, Miles City) en collaboration avec The Montana Agricultural experiment Station et les services du Gouvernement fédéral.

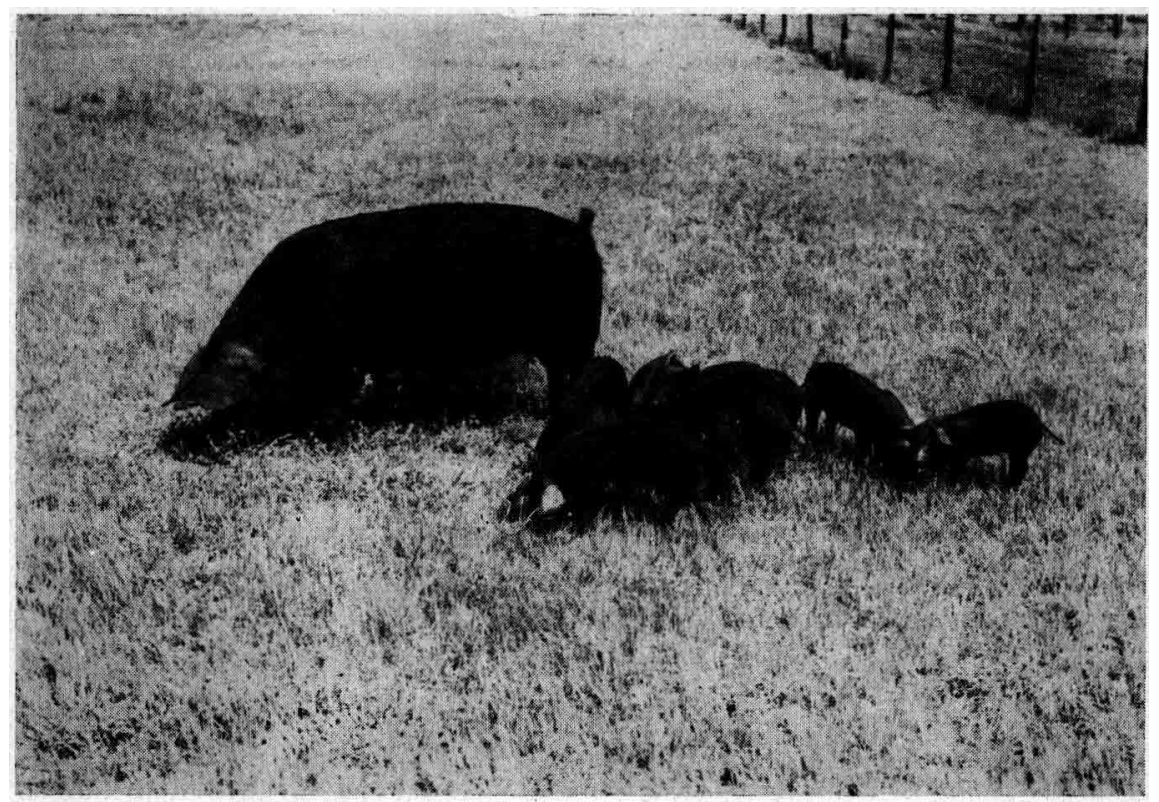

Porc Hamprace (Cliché Bureau of Animal Industry, U.S.D. A.)

La Station fédérale de Beltsville fournit $\mathrm{I} 3$ truies et 3 verrats de race danoise en 1936. On se procura par ailleurs 3 verrats et 5 truies Hampshire sans ceinture (unbelted). En r936, on procéda aux croisements :

2 verrats Hampshire $\times 5$ truies danoises, 
puis, l'année suivante, on utilisa :

2 verrats Danois et I verrat Hampshire,

5 truies danoises et 5 truies Hampshire,

avec des sujets obtenus de la I ${ }^{\text {re }}$ génération, en majorité à robe blanche. D'autres animaux de la $F_{1}$ furent croisés entre eux et, parmi les noirs obtenus, les meilleurs furent conservés pour la reproduction. Ils constituent le troupeau actuel, qui provient pour $55 \%$ du porc danois et pour $45 \%$ du Hampshire. Le coeficient de consanguiaité est de $32 \%$.

Cette race, nous l'avons indiqué, n'a pas été créée spécialement en vue de croisements ultérieurs ; on a cherché à combiner les caractères utiles des deux races d'origine, aussi bien les qualités d'élevage (fécondité, valeur laitière, etc.) que les qualités d'abattage (comme le précisent ses " auteurs": Hulton, Quensberry, Zeller Davis (28). Nous devons noter que les résultats obtenus semblent nettement supérieurs à ceux des races précédentes, celles-ci ne devant pas, rappelons-le, être en principe utilisées à l'état pur.

En I 947 , sur 45 portées :

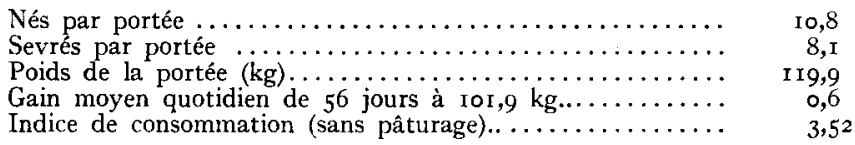

\section{LES RESUTATS}

En examinant comment ces races ont été créées, nous avons eu l'occasion de préciser leurs performances propres. Bien que, comme nous l'avons indiqué, celles-ci ne constituent pas un critère définitif (sauf pour la Hamprace) nous croyons utile de les rappeler telles qu'elles sont données par les auteurs :

\begin{tabular}{|c|c|c|}
\hline $\begin{array}{c}\text { Minnesota } \\
n^{0} \text { I }(5)\end{array}$ & $\begin{array}{l}\text { Minnesota } \\
n^{0} \quad 2\left(4^{6}\right)\end{array}$ & Hamprace (28) \\
\hline $\begin{array}{l}9,2 \\
6,8\end{array}$ & $\begin{array}{l}7,5 \\
6, \mathrm{I}\end{array}$ & $\begin{array}{r}\mathrm{ro}, 8 \\
8, \mathbf{I}\end{array}$ \\
\hline $\begin{array}{r}\mathrm{I} 2,5 \\
\end{array}$ & I 3,2 & $\mathrm{I} 4,8$ \\
\hline $7 \mathrm{I}, \mathrm{L}$ & 73,8 & 76,6 \\
\hline $\begin{array}{l}2,94 \\
\text { pâturag }\end{array}$ & $\begin{array}{l}2,94 \\
\text { Ir pâturag }\end{array}$ & $\begin{array}{ll} & 3,52 \\
\text { sans } & \text { pâturag }\end{array}$ \\
\hline
\end{tabular}

b) Carcasse :

$\%$ carcasse 5 morceaux nobles $\ldots \ldots \ldots \ldots \ldots$

$\%$ carcasse morceaux gras $\ldots \ldots \ldots \ldots \ldots \ldots$

$\begin{array}{ll}70,7 & 7 \mathbf{1}, \mathbf{1} \\ \mathbf{1} 8,6 & \mathbf{1 6 , \mathbf { I }}\end{array}$

67 env.

\section{Comparaison des races nouvelles avec les races parentes}

La première question que l'on est en droit de se poser est de savoir si, par rapport aux races originelles, les nouvelles races présentent des performances supérieures.

a) Minnesota $n^{\circ} I$.

Bien que nous n'ayons pas de précisions concernant les aptitudes des familles de porcs Tamworth et Danois utilisées et que la méthode d'appréciation préconisée par DickERSON (I6) n'ait pas été employée d'une façon systématique, nous pouvons trouver, dans la littérature, des renseignements plus généraux concernant ces races.

Pour le Tamworth DAvidson (15), indique en Angleterre : 7,2 porcelets nés par portée et 6, sevrés, mais il convient de noter que le Tamworth a été amélioré au Canada, et SMITH (49) considère les truies Tamworth comme "exceptionnellement prolifiques".

Quant au porc Danois, sa prolificité est bien supérieure ; CLAUSEN (I I) publie le tableau suivant :

\begin{tabular}{|c|c|c|c|}
\hline Années. & $\begin{array}{c}\text { Nombre } \\
\text { de portées } \\
\text { étudiées. }\end{array}$ & $\begin{array}{c}\text { Porcelets } \\
\text { nés. }\end{array}$ & $\begin{array}{c}\text { Porcelets } \\
\text { sevrés. }\end{array}$ \\
\hline $\mathbf{I} 907 \ldots$ & I 708 & Io, 6 & 8,3 \\
\hline 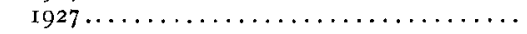 & 2815 & 10,9 & 8,4 \\
\hline $1950 \ldots \ldots \ldots \ldots \ldots \ldots \ldots \ldots \ldots \ldots \ldots \ldots \ldots$ & 3563 & II, 4 & 9,3 \\
\hline
\end{tabular}


mais l'âge à $20 \mathrm{~kg}$ est comparable à celui de nombreuses races américaines (I0) :

\begin{tabular}{|c|c|c|}
\hline & $\begin{array}{c}\text { Age } \\
\dot{\mathrm{a}} \\
20 \mathrm{~kg}\end{array}$ & $\begin{array}{c}\text { Poids à } 54 \mathrm{j} \text {. d'après la } \\
\text { méthode de WHATLEY } \\
\text { et QUAIFE (55) }\end{array}$ \\
\hline $1927 \ldots \ldots \ldots \ldots \ldots \ldots \ldots \ldots$ & $67 \mathrm{j}$ & $\mathrm{I}_{5}, 8 \mathrm{~kg}$ \\
\hline $193^{8} \ldots \ldots \ldots \ldots \ldots \ldots \ldots \ldots$ & $7 \mathrm{I} \mathbf{j}$ & $14,6 \mathrm{~kg}$ \\
\hline $1946 \ldots \ldots \ldots \ldots \ldots \ldots \ldots$ & $74 \mathrm{j}$ & $13,9 \mathrm{~kg}$ \\
\hline
\end{tabular}

En ce qui concerne la nourriture consommée, il est difficile de comparer avec exactitude des résultats américains et danois, les conditions de vie et d'alimentation étant différentes dans les deux cas (pâturage et nourriture sèche dans le nourrisseur automatique aux U. S. A., et orge + lait écrémé distribué en porcherie au Danemark). De toute façon, il faut aux porcs Danois une moyenne de $3,2 \mathrm{U}$. F. pour produire I $\mathrm{kg}$ de poids vif (Io), de 20 à $90 \mathrm{~kg}$ - alors que le Minnesota $n^{\circ}$ Iexige, au pâturage, $2,94 \mathrm{~kg}$ de mélange concentré.

Enfin, à l'abattage, il est également très difficile de comparer les mérites des deux races. Néanmoins, nous avons des points de repère : les mêmes mesures après découpage ont été effectuées par WinTers et al. (63) sur "des porcs de marché choisis ", sur des Poland China sélectionnés et sur les deux races Minnesota. Les mesures comparables donnent :

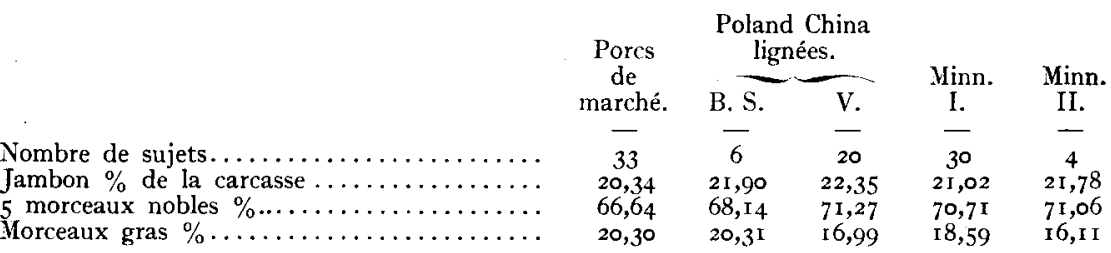

Nous pouvons ainsi constater que, sur aucun point, la race Minnesota $n^{0} I$ n'est, par ses propres performances, supérieure au Poland China sélectionné, donc, à fortiori, au Danois renommé universellèment pour sa conformation.

b) Le Minnesota $n^{0} 2$.

Les performances des souches de Poland China utilisées : Market Lady et Black Star, nous sont connues. Nous avons, d'autre part, de nombreux renseignements sur le Yorkshire Large White race qui est probablement la plus répandue dans le monde.

\begin{tabular}{|c|c|c|c|c|}
\hline & & Market & & Yorkshire \\
\hline Qualités d'abattage. & $\begin{array}{l}\text { Minn. } \\
\mathrm{n}^{\circ} 2 .\end{array}$ & $\begin{array}{c}\text { Lady } \\
(63)\end{array}$ & $\begin{array}{l}\text { Black } \\
\text { Star. }\end{array}$ & $\begin{array}{c}\text { Large White. } \\
(24)\end{array}$ \\
\hline lets nés par portée. & 7,5 & 7,2 & 7,2 & 10,57 \\
\hline g) & 13,2 & I 3, I & 12,2 & $14,9(1946)$ \\
\hline 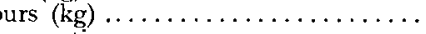 & 73,8 & 78,8 & 79,3 & $90 \mathrm{~kg}$ à $\mathrm{r} 8 \mathrm{i} \mathrm{j}$. \\
\hline tion & 2,94 & $\begin{array}{l}3,10 \\
\text { (sur p }\end{array}$ & $\begin{array}{l}3,16 \\
\text { Irage) }\end{array}$ & $\begin{array}{c}3,55 \\
\text { (en porcherie) }\end{array}$ \\
\hline
\end{tabular}

Le Minnesota $n^{\mathbf{0}} 2$ ne manifeste donc, par ses propres performances d'élevage, auc une supériorité sur les races dont il est issu, sauf l'indice.

A l'abattage, nous pouvons faire les mêmes observations que pour le Minnesota $n^{\circ} I$, quoique les carcasses lui soient supérieures.

c) Hamprace.

Nous manquons de renseignements sur les performances du Hampshire et sur les carcasses du Hamprace pour pouvoir faire d'utiles comparaisons.

Il semble que la valeur d'élevage du Hamprace soit satisfaisante et comparable à celle de races réputées, comme le Large White et le Danois. Mais rien de précis ne peut être avancé sur les carcasses. La seule comparaison possible concerne le \% de " morceaux nobles " par rapport au poids vif (28-63): le Minnesota $n^{0} I$ donne $48,9 \%$, le Minnesota $n^{0} 2$ donne $50,65 \%$ et le Hamprace, $47, \mathrm{I} \%$. Il semble donc probable que, pour la qualité de la carcasse, le Hamprace, inférieur au Minnesota $n^{0} I$, ne dépasse pas le Danois et, à fortiori, le Hampshire, qui est plus mal connu.

\section{Résultats des croisements obtenus à partir des races nouvelles}

Examinons maintenant si, utilisées comme elles doivent l'être selon leurs créateurs, les deux races Minnesota ont donné des résultats supérieurs, à la fois aux races parentes et aux autres populations porcines. 
Néanmoins, des mesures systématiques n'ont pas été effectuées, et cette lacune dans l'œuvre considérable qui a été entreprise est gênante pour conclure valablement. Nous avons rassemblé, dans le tableau suivant, l'essentiel des données dont nous pouvons disposer (63):

Réf.

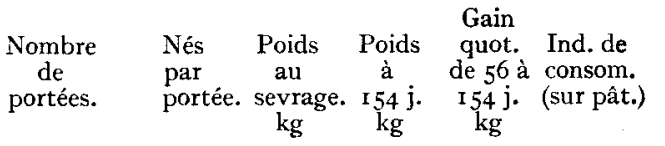

$\overline{43}$ Troupeaux d'université aux U.S. A. ... -

30 Exp. d'alimentation............... 79 exp.

3o Minn. $n^{\circ}$ I $x n^{0} 2 \ldots \ldots \ldots \ldots \ldots$

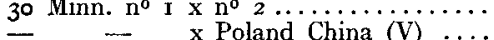

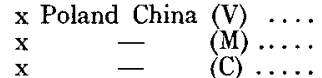

$\mathrm{x} \mathrm{V} \times$ Minn $\mathrm{n}^{\circ}$

$\mathrm{x} M \ldots \ldots \ldots \ldots \ldots \ldots \ldots \ldots$

$x \vee \ldots \ldots \ldots \ldots \ldots \ldots$

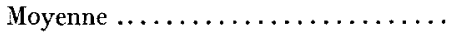

Minnesota $n^{\circ}$ I seul

Minnesota $n^{\circ} 2$ seul ............

8
I 5
I
3
I
7
9

(5,5 sevrés) $\overline{\mathrm{I} 2,2}$

$\begin{array}{rrrrr}7,4 & -12,2 & 72,9 & 0,64 & - \\ 18,3 & 93,3 & 0,76 & 3,16\end{array}$

$\begin{array}{lllll}7,4 & 17,3 & 93,3 & 0,76 & 3,16 \\ 7,7 & 17,8 & 91,0 & 0,75 & 3, \text { I9 }\end{array}$

$\begin{array}{lllll}5,9 & 19,0 & 87,9 & 0,70 & 3,33\end{array}$

$\begin{array}{lllll}5,0 & 18,8 & 88,8 & 0,7 \mathrm{I} & 3,41\end{array}$

$8,5 \quad$ I $7,4 \quad 82,9 \quad 0,66 \quad 3,43$

$8,5 \quad$ I $7,4 \quad 82,9 \quad 0,66 \quad 3,43$

$\begin{array}{lllll}7,4 & \mathrm{I} 7,5 & 84,3 & 0,67 & 3,00\end{array}$

$6,99 \quad I 7,7 \quad 87,4 \quad 0,7$ I $\quad 3,24$

$9,2(6,8$ sev. $)$ I $2,5 \quad 7 \mathrm{I}, \mathrm{I} \quad-\quad 2,94$

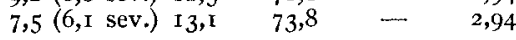

D'une façon générale, il semble que les animaux croisés sont supérieurs aux parents en ce qui concerne le poids au sevrage, la vitesse de croissance, mais non pour la prolificité. Quant à la comparaison avec les troupeaux d'université ou les résultats d'expériences d'alimen tation, elle nous paraît peu probante, ces performances étant nettement inférieures aux moyennes générales des U.S. A.

Quoi qu'il en soit, les résultats les plus favorables obtenus à la suite de ces croisements sont généralement inférieurs ou ne dépassent pas très sensiblement les résultats observés chez les races européennes perfectionnées, comme le Danois ou le Large White. Seule la précocité des croisements Minn. $n^{0} I \times$ Minn. $n^{0}{ }_{2}$, Minn. $n^{\circ} \times V$, Minn. $n^{0}$ I $\times M$, nous paraît remarquable.

Les comparaisons des carcasses sont également données (63). Nous en reproduisons cidessous l'essentiel. (Nous appelons : le Minnesota $n^{\circ} I=1$; le Minnesota $n^{\circ} 2=2$, et les différentes souches de Poland China par leur initiale.)

\begin{tabular}{|c|c|c|c|c|c|c|c|}
\hline Origine. & "C & $\begin{array}{l}\text { arce } \\
" .\end{array}$ & $\mathrm{IxV}$. & $\mathrm{I}\left(\mathrm{IxV}^{-}\right.$. & $\begin{array}{c}\mathrm{VxI} \\
(\mathrm{IxV}) .\end{array}$ & IxM. & I $(\mathrm{Ix})$ \\
\hline Iombre de carcasses étudi & & & & & 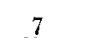 & 23 & 8 \\
\hline ment $\% \ldots . . .$. & $\begin{array}{l}53 \\
69,80\end{array}$ & & & & 68,37 & 68,95 & 67,92 \\
\hline de la & 76,7 & & 77 & 77 & 78,0 & 77,0 & \\
\hline et. . & & & & & 21,08 & & $2 \mathrm{I}, \mathrm{I} 5$ \\
\hline bles $\% \mathrm{p}$ & 66,64 & & & 71 & 70,99 & 67,82 & 71,05 \\
\hline$\% \%$ & 20,30 & & 19,33 & I & 17,60 & 20,27 & I 7 \\
\hline Origi & $(\mathrm{Lx}$ & & $2 \times M$ & $(2 \times M)$ & $2 \times V$ & $V(2 \times V)$ & $2 \mathrm{xML}$ \\
\hline usses & & & & 24 & 6 & 6 & 2 \\
\hline . & 68,65 & 83 & $68,8 \mathrm{I}$ & 69,27 & 69,42 & 67,49 & 74,20 \\
\hline de la carcasse & $7^{6,2}$ & $78, \circ$ & 76,7 & 74,9 & 75,9 & & 80,0 \\
\hline s net & 21,72 & 69 & 21,58 & 8 & 21,75 & 3 & 23,00 \\
\hline $\operatorname{les} \%$ & 70,46 & & 71,19 & & 71,76 & Io & 70,04 \\
\hline$\%$ & & & 16,04 & 16,36 & I 5,54 & 16,23 & $I 7,21$ \\
\hline Ori & $2 \times \mathrm{B}$ & $2 x$ & $2(2 \times I)$ & $2(\mathrm{VxI})$ & $\mathrm{V}(2 \times I)$ & $\begin{array}{c}\mathrm{Ix2} \\
(\mathrm{VxI})\end{array}$ & $\frac{V \times I}{2(V x I)}$ \\
\hline & - & - & - & - & - & - & - \\
\hline asses & I & & & & 4 & & 2 \\
\hline . & $\begin{array}{l}71,65 \\
81,3\end{array}$ & & $\begin{array}{l}66,62 \\
74,9\end{array}$ & & 9 & 7 & 77,7 \\
\hline & 21,6 & & 21,40 & & 78 & & 03 \\
\hline 4 & & & 72,17 & & & & \\
\hline Morceaux gras \% & 18,55 & I 8, OI & $17,3^{1}$ & 16,27 & 18,39 & 18,98 & 16,18 \\
\hline
\end{tabular}

On peut constater, pour certains croisements, une amélioration sensible par rapport aux parents et surtout par rapport aux porcs courants, en ce qui concerne :

le pourcentage des cinq morceaux nobles,

le rendement en viande nette, 
le pourcentage de morceaux gras.

Le pourcentage du poids du jambon ne varie pas beaucoup.

Mais nous devons noter:

$\mathrm{I}^{0}$ que les performances les plus intéressantes $(2 \times \mathrm{ML}), 2(2 \times \mathrm{I}), \mathrm{V}(2 \times \mathrm{I}),(\mathrm{V} \times \mathrm{I}) 2(\mathrm{~V} \times \mathrm{I})$, concernent un nombre d'échantillons réduits, ce qui leur ôte une grosse partie de leur signification ;

$2^{\circ}$ que les porcs courants américains servant de comparaison ont une conformation tout à fait défectueuse comparée à certaines races européennes, bien que les critères d'appréciation soient généralement les mêmes en France et aux U. S. A. Nous avons eu l'occasion (2I) de voir à la Station fédérale de Beltsville deux carcasses correspondant, l'une à la qualité courante du commerce (Poland China), l'autre à la qualité " extra "U.S. A. provenant d'un croisement (Poland China $\times$ Danois). Le pourcentage des cinq morceaux nobles était, respectivement, par rapport au poids vif, de 46,8 et $5 \mathrm{I}, 9 \%$ (nous avons vu précédemment : Minn. $\mathrm{n}^{0}$ I : 48,9 - Minn. $\mathrm{n}^{\mathrm{0}} 2: 5^{\circ}, 65$ - Hamprace : 47, I - croisement $(\mathrm{V} \times \mathrm{I}) 2(\mathrm{~V} \times \mathrm{I}): 50$ - porc du commerce $: 46,5)$. Or, ce porc dont la carcasse nous a été présentée comme un succès, avait une couche de lard dorsal de $36,2 \mathrm{~mm}(52,8 \mathrm{~mm}$ pour l'autre), alors que les porcs Large White français couramment abattus à la Station du porc de Bois-Corbon (34) ont une épaisseur de lard : au dos... $26 \mathrm{~mm}$; au rein... $30 \mathrm{~mm}$.

\section{CONCL USION}

L'œuvre réalisée par les zootechniciens américains présente un intérêt certain, car elle démontre qu'il est possible de constituer un troupeau porcin fermé sans que la consanguinité ne provoque de graves dommages, et de créer une race en un temps incomparablement plus court qu'avec les méthodes traditionnelles. Ainsi, devient réalisable l'idée séduisante de croiser avec profit des lignées "épurées" par la consanguinité.

Mais en ce qui concerne l'application pratique des résultats obtenus jusqu'à présent, il nous paraît que ceux-si sont quelque peu décevants dans l'état actuel des choses, puisque, dans l'ensemble, les performances observées sur des sujets élevés dans des conditions satisfaisantes, si elles montrent un net progrès par rapport à la plupart des races américaines, ne sont pas supérieures à celles que l'on observe dans des races plus anciennes et sélectionnées par les méthodes classiques, qui par ailleurs conservent une grande rusticité. Les chiffres donnés par les chercheurs eux-mêmes sont loin de nous porter, comme VOEGELI (53) à un sentiment systématiquement admiratif.

Nous ne saurions cependant voir là un motif de condamner une méthode trop récente pour être déjà jugée à ses résultats et qui peut, un jour; se révéler extrêmement féconde ; mais nous pensons qu'à l'heure actuelle l'exploitation, du moins en Europe. de ces nouvelles races présente un intérêt qui nous paraît discutable.

\section{BIBLIOGRAPHIE}

(1) Allix (J.). -- 195I. Ann. de la Nutrition et de l'Alimentation, V, I, II3-I45.

(2) ALLIX (J.). - I95I. Revue du Ministere de l'Agriculture, 7, 167-175.

(3) BERGE (S.). - 1946. Innavl. og Smägrisd ødlighet. Tidsskr. norske Landbr., 53, 305-315.

(4) Briggs (H. M.). - 1943. Modern breeds of livestock. McMillan $\mathrm{C}^{\boldsymbol{o}}$, N. Y.

(5) Brugman (II. H.), Winters (L. M.), Dailey (D. L.). - i949. A genealogy study of the Minnesota no r. Minn. Agr. Exp. Sta. Tech. Bul.. I84.

(6) Carroll (W. E.), Roberts (E.). - 1942. Crossbreeding in swine. Illinois Agr. Exp. Sta. Bul., 489 .

(7) Carroll (W. E.), Krider (I. L.). - i95i. Swine production. McCraw Hill Book $\mathrm{C}^{\circ}$.

(8) Chambers (D.). - 1950. J. Animal Science, 9, 4, 635.

(9) Chambers (D.), Whatley (J. A.). - I95I. Heterosis in crosses of inbred lines of Duroc swine. J. Animal Science, 10, 2, 505.

(то) Clausen (H. J.). - I946-47. $36^{\mathrm{e}}$ Beretning om Sammenlignen de Forsøg med Svin fra Statsanerkendte Avlscentre. $233^{\mathrm{e}}$ Beretning fra Forsglaboratoriet.

(i r) Clausen (H. J.). - I95 I. Die Mastleistungs Prùfungen und deren Erfolg fùr die Futterung verwertung und die typnäszge Entwicklung der dänischen Schweinrasse. Züchtungskunde, 22, 5,204 .

(12) Comstock (R. E.), Winters (L. M.). - i943. A comparison of the effects of inbreeding and selection on performance in swine. J. An. Sci., 2, 4, 356 . 
(I3) CRAft (W. A.). - - 943 . Swine breeding research at the regional swine breeding laboratory. U. S. Dept. Agr. Misc. Public., $\mathrm{n}^{0} 523$.

(14) Crowther. - I945. British Soc. of Animal Production. Meeting, 21.2.45.

(I5) Davidson (H. R.). - I948. The production and marketing of pigs. Longmans, Green et $\mathrm{C}^{\circ}$, London.

(16) Dickerson (G. E.). - I942. Experimental design for testing inbred lines of swine J. An. Sci., 1, 4, 326 .

(1 7) Dickerson (G. E.), Lush (J. L.), Culbertson (C. C.). - I946. Hybrid vigor in single crosses between inbred line of Poland China swine. J. An. Sci., 5, I, I6.

(i 8) Dickerson (G. E.), Lush (J. L.), Baker (M. L.), Whatley (J. A.), Winters (L. M.). - I947. Performance of inbred lines and line crosses in swine. J. An. Sci., 6, 4, 477.

(ig) Dickerson (G. E.). - r951. Effectiveness of selection for economic characters in swine. J. Ah. Sci., 10, I, 12.

(20) Durham (R. M.), Chapman (A. B.), Grummer (R. H.). - ig49. Inbred and linecross boars versus non inbred and non linecross boars used in two-sire herds on Wisconsin farms. J. An. Sci., 8, 4, 605 .

(21) FÉvRIER (R.). - I950. Rapport sur mission aux U. S. A. non publié.

(22) GodBEy (E. G.), STARKEY (L. V.). - 1932. A genetic study of the effects of intensively inbreeding Berkshire swine. S. C. Agr. Exp. Sta., Ann. Rep.

(23) Hazel (L. N.), Husson (A. L.), Lush (J. L.). - 1948. Comparisons of inbred Poland China, Landrace and purebred boars on Iowa farms. J. An. Sci., 7, 4, 512.

(24) Herd-Book du Porc Large White: Le Porc Large White, Paris.

(25) Hetzer (H. O.), Harkins (O. G.), Zelier (T. H.). - 1950. Performance of crosses between six inbred lines of swine. $J . A n$. Sci., 9, 4, 638 .

(26) HodGson (R. E.). - 1935. An eight generation experiment in inbreeding swine. $J$. Heredity, 26, 5, 209.

(27) Hughes (E. H.). - 1933 . Inbreeding Berkshire swine. J. Heredity, 24, 5, 133.

(28) Hutton (R. E.), Quensberry (J. R.), Zeller (J. H.), Davis (R. L.). - i 948 . The Hamprace hog. Montana Agr. Exp. Sta., Bul. 454.

(29). JoHANSSON (I.). - Svenska Svinavelsföreningens tidskrift, 2-3.

(30) KING (H. D.). - I919. Studies on inbreeding. IV. J. Exp. Biol., 29, 71.

(3I) Kottman (R. M.), Lush (J. L.), Hazel (L. N.). - 1948. Selection in inbred lines of swine. J. An. Sci., \%, 4, 513.

(32) Lambert (W. V.). - I940. Amer. Soc. An. Prod., Proc., i940, 378.

(33) Le Conte (J.). - IIe Congrès international du Maïs, I2 I-I35.

(34) Leroy (A. M.), Février (R.). - ig49. Etude des aptitudes économiques et de leur transmission chez les différentes souches et races porcines. Ann. Agron., $\mathrm{n}^{\circ} 6$.

(35) Lush (J. L.). - I95r. Genetics in the 2oth Century. McMillan Co, N. Y.

(36) Lush (J. L.), Culbertson (C. C.). - 1937. Consequence of inbreeding Poland China hogs. Iowa Agr. Exp. Sta. Ann. Rep., p. 80.

(37) LUSh (J. L.), Molla (A. E.). - r942. Litter size and weight as permanent characteristics of sows. U.S. D. A. Tech. Bul., 836 .

(38) Lush (J. L.), Stearer (P. S.), Culbertson (C. C.). - ig39. Crossbreeding hogs for pork production. Iowa Agr. Exp. Sta. Bul., 38o.

(39) Lush (J. L.), Culbertson (C. C.), Craft (W. A.), Stearer (P. S.), Hazel (L. N.). 1948. Rép. Agr. Res. for the year ending June 30, 1948, I 7-32.

(40) McPhee (H. C.), Russel (E. Z.), Hugh (C.), ZeLleR (J. H.). - 1931. An inbreeding experiment with Poland China swine. J. Heredity, 22, I2, 393.

(4I) National Pig Breeders' Association. Large White pigs, London.

(42) QuitTeT (E.). - 1946. Les races bovines françaises. Ed. Minis. Agric.

(43) Roberts (E.), Carroll (W. E.). - 1939. A study of hybrid vigor in a cross between Poland China and Duroc Jersey swine. J. Agr. Res., 59, 847-854.

(44) Robison (W. L.). - I946. Crossbreeding for the production of market hogs. Ohio Agr. Exp. Sta., Bul. 242, vol. 31.

(45) RoBison (W. L.). - 1948. Crossbreeding for the production of market hogs. Ohio Agr. Exp. Sta., Bul., 675 .

(46) RoubiceK (C. B.), WiNTERS (L. M.), Kiser (O. M.). - I95I. A genealogical study of the Minnesota $\mathrm{n}^{\mathbf{0}}{ }_{2}$ hog. Minnesota Agr. Exp. Sta. Tech. Bul., I33.

(47) Sierk (C. F.). - 1948. A study of heterosis in swine. J. An. Sci., \%, 4, 515.

(48) SIERK (C. F.), Winters (L. M.). - 195I. A study of heterosis in swine. J. An. Sci., 10, I, 104 .

(49) SMITH (W. W.). - I937. Porc production. McMillan Co, N. Y.

(5o) Stewart (H. A.), Comstock (R. E.). - I948. A comparison of inbred lines of swine based on their performance in topcrosses. J. An. Sci., y, 4, 515. 
(5I) Stringam (E. W.), Winters (L. M.), Jordan (P. S.). - I95o. The genealogy of an inbred Poland China herd of swine. Minnesota Agr. Exp. Sta. Tech. Bul., Igr.

(52) U. S. Dept. Agr. Prod. and marketing adm. - Statistical Bul., 91, 1950.

(53) Voegeli (S.). - I 951 . Nuove razze suine ottenute in America con i metodi della genetica. Rivista di Zootecnia, XXV, 7, 235.

(54) Warwick (E. J.), WILEY (J. R.). - I949. Growth rates of some inbred, purebred, crossbred and crossline pigs. J. An. Sci., 8, 4, 6ro.

(55) Whatley (J. A.), Quaife (E. L.). - 1937. Adjusting weight of pigs to a standard age of 56 days. Amer. Soc. An. Prod. Proc.

(56) Whiteman (J. V.), Hillier (J. C.), Whatley (J. A.). - 195I. Carcass studies on hog of differend breeding. J. An. Sci., 10, 3, 638 .

(57) WILHAM (O. S.), CRAFT (W. A.). - I939. An experimental study of inbreeding and outbreeding in swine. Oklahoma Agr. Exp. Sta. Tech. Bul., 7.

(58) Winters (L. M.), K ISer (O. M.), Jordan (P. S.). Peters (W. H.), - I935. A six years study of crossbreeding swine. Minnesota Agr. Exp. Sta. Bul., 32o.

(59) Winters (L. M.), Winters (O. M.), Jordan (P. S.), Peters (W. I..). - 1936. Crossbred swine. Minnesota Agr. Exp. Sta. Spec. Bul., r80.

(60) Winters (L. M.), Comstock (R. E.), Dailey (D. C.). - i943. The development of an inbred line of swine from a crossbred foundation. $J$. An. Sci., 2, 2, I29.

(6r) Winters (L. M.), Comstock (R. E.), Hodgson (R. E.), Kiser (O. M.), Jordan (P. S.). DAILEY (D. L.), - I943. Experiments with inbreeding swine and sheep. Minnesota Agr. Exp. Sta. Bul., 364 .

(62) Winters (L. M.), Cummings (J. N.), Stewart (H. A.). - I947. A study of factors affecting survival from birth to weaning and total weaning weight of the litter in swine. J. An. Sci., 6, 3, 288.

(63) Winters (L. M.), Dailey (D. L.), Jordan (P. S.), Kiser (O. M.), Hodgson (R. E.). Cummings (J. N.), Sierk (C. F.). - I948. Experiments with inbreeding swine. Minnesota Agr. Exp. Sta. Bul., 400.

(64) WRIGHT (S.). - 1292. The effect of inbreeding and crossbreeding on guinea pigs. U. S. Dept. Agr. Bul., I090-I I2I.

(65) WRIGHT (S.). - I921. Sytèms of mating. Genetics, 6, I II-I 78.

(66) WRIGHT (S.). - I I22. Coefficient of inbreeding and relationship. Amer. Nat., 56, 330338.

Le Directeur-Gérant : B. LACLAVIÈRE. 